\title{
REVIEW
}

\section{Effects of obstructive sleep apnoea on heart rhythm}

\author{
Valentina A. Rossi*, John R. Stradling ${ }^{\#}$ and Malcolm Kohler*,
}

ABSTRACT: Symptomatic obstructive sleep apnoea (OSA) has been proven to be a risk factor for hypertension and vascular dysfunction, and has been proposed to be causally related with cardiac arrhythmias and sudden cardiac death.

Searches of bibliographical databases revealed that several mechanisms seem to underpin the association between OSA and cardiac arrhythmias: intermittent hypoxia associated with autonomic nervous system activation and increased oxidative stress, which may lead to cardiac cellular damage and alteration in myocardial excitability; recurrent arousals, resulting in sympathetic activation and coronary vasoconstriction; and increased negative intrathoracic pressure which may mechanically stretch the myocardial walls and, thus, promote acute changes in myocardial excitability as well as structural remodelling of the myocardium.

Findings from cross-sectional studies suggest a high prevalence of cardiac arrhythmias in patients with OSA and a high prevalence of OSA in those with cardiac arrhythmias. Preliminary evidence from uncontrolled interventional studies suggests that treatment of OSA may prevent cardiac arrhythmias.

In conclusion, there is preliminary evidence that OSA is associated with the development of cardiac arrhythmias. Data from randomised controlled studies are needed to definitively clarify the role of OSA in arrhythmogenesis.

KEYWORDS: Atrial fibrillation, autonomic nervous system, cardiac arrhythmias, hypoxaemia, sleep disordered breathing, sudden cardiac death

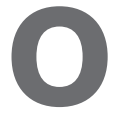
bstructive sleep apnoea (OSA) is a common sleep-related breathing disorder estimated to affect between $5 \%$ and $56 \%$ of middle-aged men in western countries, depending on the exact definition of OSA and the prevalence of obesity [1,2]. OSA is characterised by transient mechanic interruption of the airflow through the upper airways which can be complete (apnoea) or partial (hypopnoea). Apnoea or hypopnoea lead to oxygen desaturations, increased inspiratory effort, arousals from sleep and, as a consequence, to increased daytime sleepiness [3].

Symptomatic obstructive sleep apnoea syndrome (OSAS) has been proven to be a risk factor for hypertension, heart failure and vascular dysfunction, and has been proposed to be causally related to both non-fatal and fatal coronary and cerebrovascular events. Treatment of patients with OSA with continuous positive airway pressure (CPAP) seems to reduce mortality from cerebral and myocardial events [4-7].

Preliminary data suggest that there is also a relationship between sleep-disordered breathing, cardiac arrhythmias and sudden cardiac death [8]. OSA has mainly been associated with premature atrial complex short runs, sinus bradycardia, sinus pauses, premature ventricular complexes and paroxysmal atrial fibrillation [9-11], while central sleep apnoea (CSA) has mainly been associated with atrial fibrillation [12]. There is also evidence for a close temporal relationship between arrhythmia and obstructive apnoeas [13].

The aim of this review is to describe the pathophysiological mechanisms underlying the association between OSA and cardiac arrhythmias, and provide an overview on the available evidence from clinical studies on this relationship. The possible association between CSA and

\section{AFFILIATIONS}

*Sleep Disorders Centre and Pulmonary Division, University Hospital of Zurich, Zurich, and

"Zurich Centre for Integrative Human Physiology, University of Zurich, Zurich, Switzerland.

${ }^{\#} 0 x$ ford Centre for Respiratory Medicine, Churchill Hospital, Oxford, UK.

CORRESPONDENCE

M. Kohler

Sleep Disorders Centre and

Pulmonary Division

University Hospital Zurich

Raemistrasse 100

8091 Zurich

Switzerland

E-mail: Malcolm.K@bluewin.ch

Received

Aug 152012

Accepted after revision:

Dec 022012

First published online:

Dec 202012 
cardiac arrhythmia, however, will not be addressed in this review.

\section{SEARCH AND REVIEW METHODS FOR IDENTIFICATION OF STUDIES}

Studies concerned with cardiac arrhythmia and OSA were identified by searches of bibliographic databases including PubMed and manual searching of respiratory and cardiology journals. All abstracts found in the databases were assessed by two authors (V.A. Rossi and M. Kohler) to determine their potential relevance for full review. Subsequently the full text of the studies was reviewed.

\section{MECHANISMS POTENTIALLY UNDERPINNING THE ASSOCIATION BETWEEN OSA AND CARDIAC ARRHYTHMIAS}

Several pathophysiological consequences of OSA, including intermittent hypoxia-induced oxidative stress, recurrent arousals and intrathoracic pressure swings may provoke cardiac arrhythmias (fig. 1), either directly or via effects on the autonomic nervous system. In the following section, these mechanisms will be discussed separately with a focus on the effects on the autonomic nervous system.

\section{Intermittent hypoxia}

In OSA, recurrent apnoea and hypopnoea lead to repeated and marked arterial oxygen desaturations which cyclically recur during the night. Although it has not been established whether it is the hypoxaemic phase, or the following re-oxygenation phase, that exerts the most damage, intermittent hypoxia has been

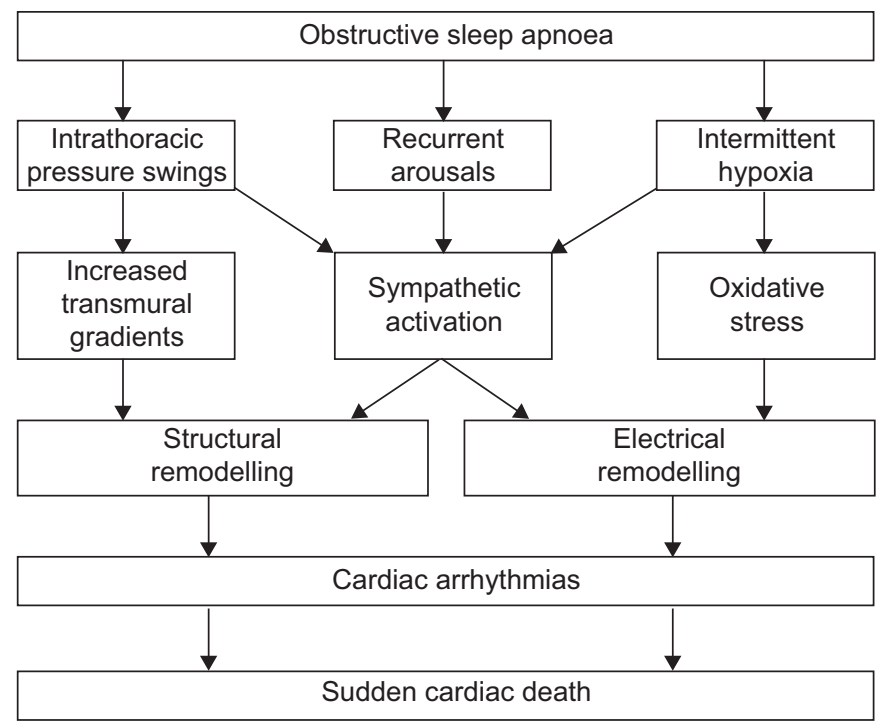

FIGURE 1. Possible mechanisms underpinning the association between obstructive sleep apnoea and cardiac arrhythmias. Three main mechanisms are thought to trigger atrial and ventricular arrhythmias. Intrathoracic pressure swings are associated with increased transmural gradients, exerting tension forces which might lead to structural remodelling of the cardiac walls. Sympathetic activation induced by recurrent arousals, intermittent hypoxia, oxidative stress and intrathoracic pressure swings is thought to induce electrical remodelling and electrical instability in the myocardium. Structural and electrical remodelling represents the substrate on which dysrhythmias onset. Increased oxidative stress may also provoke electrical remodelling and thus increase the risk of arrhythmias. proposed to act by inducing release of reactive oxygen species (ROS) and by increasing sympathetic nerve activity [14-16].

\section{Intermittent hypoxia and the autonomic nervous system}

Under physiological conditions hypoxaemia acts on the carotid body by promoting both hyperventilation and sympathetic activation [14, 15]. In contrast, upper airway obstruction, by preventing lung expansion and stretching of vagolytic fibres in the lung, elicits the diving reflex (increased sympathetic vasoconstriction to muscles and viscera in order to maintain oxygen delivery to the vital organs [9]). This leads to a rise in blood pressure and vagally induced reflex bradycardia [14, 15]. The resultant hyperpnoea on arousal stretches the peripheral afferent fibres in the lung, which is associated with a vagolytic response (Herning-Breuer reflex) and, along with arousalrelated increases in sympathetic tone, greatly increase heart rate $[14,15]$. Therefore, the responses to obstructive apnoea include both an increase in the sympathetic and the parasympathetic tone to the heart and peripheral vasculature.

In rodents, exposure to intermittent hypoxia leads to an increased density of noradrenergic terminals in the trigeminal sensory and motor nuclei [17]. However, this mechanism also results in maladaptive changes, such as an increase in the noradrenergic pathways within the brainstem, thereby activating the medullary region [17]. Both the activation of the medullary region, and the direct stimulation of the carotid bodies by intermittent hypoxia, lead to increased sympathetic nervous system activity and enhance catecholamine secretion from adrenal medullary chromaffin cells [18, 19].

Hypoxaemia has been suggested to induce both bradycardic and tachycardic arrhythmias. In dogs, hypoxaemic stimulation of the carotid bodies causes bradycardic arrhythmias via vagal activation and this reflex can be prevented by atropine [15]; while tachycardic arrhythmias are due to sympathetic activation and can be prevented by ganglionated plexis ablation or autonomic blockade [14, 15, 20].

Patients with OSA have a greater sympathetic nerve activity during sleep and wakefulness when compared with controls, and as a consequence an elevated heart rate and diurnal hypertension (as well as non-dipping status) are commonly observed in these patients [21, 22]. Continuous sympathetic stimuli repeated during each apnoea may promote carotid chemoreflex and increase the sympathetic drive resulting in increased heart rate and rises in systolic blood pressure at the end of each apnoeic event [22]. Evidence from cross-sectional studies in humans suggests an increase in arrhythmias in relation to the number and degree of oxygen desaturations registered during sleep [23-26]. There is ample evidence from randomised controlled trials proving that treatment of OSA with CPAP reduces sympathetic nervous system activity [27, 28].

\section{Intermittent hypoxia and oxidative stress}

In vitro studies have demonstrated that intermittent cycles of hypoxia and re-oxygenation are associated with increased oxidative stress, due to generation of ROS resulting from the inhibition of complex I of the mitochondrial electron transport chain, increased activity of reduced NADPH and xanthine oxidases, and decreased levels of antioxidants [16, 29, 30]. Findings from animal studies have shown that oxidative stress 
induced by intermittent hypoxia leads to protein oxidation and myocardial lipid peroxidation [31, 32]. Rodents exposed to intermittent hypoxia develop significant myocardial cell injury, such as myocyte hypertrophy, increased myocyte cell length and cell apoptosis as evidenced by rises in serum cardiac troponin I [33, 34]. Moreover, evidence of left ventricular dysfunction as assessed by increased left ventricular end-diastolic pressure has been found in rats exposed to intermittent hypoxia, in which the decrease in left ventricular function was related to the increase in lipid peroxides [31]. Such structural alterations of the myocardium may lead to micro-ischaemia, promoting cardiac repolarisation abnormalities and thus increased susceptibility to develop ventricular dysrhythmias [35].

In humans, augmented oxidative stress has been suggested to increase the risk of cardiac arrhythmia by: depletion of ATP levels, oscillations in the mitochondrial membrane potential, and changes in matrix concentrations of $\mathrm{Ca}^{2+}, \mathrm{K}^{+}, \mathrm{NADH}, \mathrm{ADP}$ and tricarboxylic acid cycle intermediates [36]. These alterations create myocardial regions of inhomogeneous myocardial excitability favouring the development of re-entry arrhythmias [36, 37].

In addition, ROS may not only have a direct effect on intracellular structures, but also promote the sympathetic response in carotid bodies and the adrenal medulla's production of catecholamines in response to hypoxia $[19,38]$.

In patients with OSA, CPAP therapy has been proven to reduce markers of oxidative stress in a randomised-controlled trial [39].

\section{Arousals}

In OSA, as a consequence of the interruption of ventilation, blood oxygen levels decrease while carbon dioxide levels rise and inspiratory effort increases. Depending on the individual threshold, this may lead to arousals from sleep in order to restore a normal breathing pattern and normalise blood gases.

\section{Arousals and the autonomic nervous system}

In animal models of OSA, experimentally simulated obstructive apnoea led to arousals which were associated with an increase in sympathetic activity. In dogs, spontaneous arousals from sleep were associated with acute rises in heart rate due to both sympathetic activation and parasympathetic withdrawal, and could be prevented by sympathetic block [40]. In pigs, arousals induced by tracheal obstruction during sleep were associated with increases in blood pressure, heart rate and coronary vascular resistance [41]. $\alpha$-Adrenergic receptor blockade eliminated increases in blood pressure and decreased coronary vascular resistance [41].

In healthy subjects, bursts of sympathetic nerve activity and increases in blood pressure were recorded in response to arousal stimuli during sleep [42]. In patients with OSA, arousals occurring at the end of apnoeas and hypopnoeas are associated with marked transient increases in sympathetic nervous activity which led to considerable blood pressure rises [43]. Moreover, by acute hyperactivation of the sympathetic nervous system, arousals provoked coronary vasoconstriction [44] resulting in micro-ischaemia, which may increase dispersion of, and prolong, myocardial repolarisation [45]. In a casecontrol study, including patients with OSA and snorers and healthy subjects as a control, ST-segment depression in the electrocardiogram (ECG) as a surrogate of ventricular microischaemia was observed in patients with OSA but not in controls [46]. In the latter study, daytime and nocturnal STsegment depression episodes were related to the arousal index and increased daytime urinary epinephrine excretion [46].

\section{Increased negative intrathoracic pressure}

Negative intrathoracic pressure and increased transmural gradients

Obstructive apnoea and hypopnoea are associated with repeated inspiratory efforts against the collapsed upper airways producing considerable negative intrathoracic pressure, which may be as low as $-80 \mathrm{mmHg}$. This mechanism, repeated during each apnoeic phase, may stretch the cardiac wall and intrathoracic vessels possibly leading to both shortterm electrical and long-term mechanical remodelling of both atria and the left ventricle, thereby increasing the risk for the onset of atrial and ventricular dysrhythmias [47, 48].

In vitro and in vivo studies on myocardial tissue have provided evidence for acute stretch-activated $\mathrm{Ca}^{2+}$ channels which produce functionally significant repolarisation gradients and promote both early and delayed after-depolarisations, thereby predisposing to ventricular arrhythmias [49, 50]. Similar results were found in an animal model, in which negative intrathoracic pressure during obstructive respiratory events induced shortening of the right atrial refractory period and consequently increased the susceptibility to premature beats and atrial fibrillation [51].

In humans, the Mueller manoeuvre, an inspiratory effort made against a closed mouthpiece and nose, has been proven to be an adequate way to induce intrathoracic pressure changes resembling those observed during an obstructive apnoea; but without the confounding effects of hypoxaemia, arousals from sleep, and comorbidities often present in patients with OSA. During the Mueller manoeuvre, the negative intrathoracic pressure transmitted to the pericardial cavity leads to an increase in the left ventricular afterload, in the pressure developed during the left ventricular isovolumetric phase, and in left ventricular volume [52, 53]. In healthy subjects, simulated obstructive apnoea or hypopnoeas were associated with an acute increase in proximal aortic diameter and in left ventricular volume, as assessed by echocardiography, while left atrial volume and left ventricular ejection fraction were reduced [53-55]. These findings suggest that repeated intrathoracic pressure changes in OSA might play a role in the onset of cardiac arrhythmias by promoting both mechanical and electrical remodelling of the heart, as evidenced by recent findings [48].

\section{Negative intrathoracic pressure and the autonomic nervous system}

In an animal model of OSA, negative tracheal pressure leads to a pronounced shortening of the atrial effective refractory period and increased atrial fibrillation inducibility, which are mediated by sympathetic $\alpha$ - and $\beta$-pathways, vagal activation and ganglionated plexus [56].

In humans, the negative intrathoracic pressure provoked by the Mueller manoeuvre is associated with a substantial rise, of 
more than $200 \%$, in postganglionic sympathetic nerve activity leading to a significant increase in mean blood pressure at the end of the apnoea [57].

In summary, the increased susceptibility to arrhythmia in patients with OSA may primarily be due to both structural and electrical remodelling of the heart over time, which are promoted by excessive negative intrathoracic pressure and increased sympathetic activation, and thus provide an arrhythmogenic substrate for the acute triggers resulting from apnoea and hypopnoea.

\section{CURRENT EVIDENCE FROM CLINICAL STUDIES ON THE ASSOCIATION BETWEEN OSA AND CARDIAC ARRHYTHMIAS}

In the following sections, the evidence from observational and interventional studies on the association between OSA and cardiac arrhythmias will be discussed.

\section{Atrial fibrillation and atrial arrhythmias}

The most frequent atrial arrhythmias include atrial fibrillation, supraventricular premature complex (SVPC), and supraventricular tachycardia (SVT).

Patients with OSA have been shown to have electrical abnormalities in electrophysiological mapping studies, such as longer P-wave duration, prolonged conduction times and longer sinus node recovery, as well as mechanical alterations, such as atrial enlargement and inter-atrial and intra-atrial electromechanical delay, compared with control subjects [48, 58]. These observations suggest that patients with OSA may have an increased susceptibility to develop atrial fibrillation. In addition, atrial fibrillation development has been proposed to be related to hypoxaemia, since the number of nocturnal oxygen desaturations (ODI) has been found to be an independent risk factor for atrial fibrillation and lower nocturnal oxygen saturation is associated with higher recurrence of atrial fibrillation after initially successful cardioversion [59].

\section{Observational studies regarding atrial fibrillation}

The prevalence of nocturnal atrial fibrillation in patients with OSA has been estimated to be between $3 \%$ and $5 \%$, compared with a prevalence of between $0.4 \%$ and $1 \%$ in the general population or control subjects without OSA $[10,23,60]$.

In an observational study, including obese patients, the 5-year incidence of atrial fibrillation in patients with OSA has been estimated to be $4.3 \%$ compared to a $2.1 \%$ incidence in obese patients without OSA, thus resulting in a hazard ratio for atrial fibrillation of 2.2 in patients with OSA [59].

The prevalence of atrial fibrillation has also been found to be higher in patients with coronary artery disease (CAD) and OSA, compared to patients with CAD but without OSA $(32 \%$ and $18 \%$, respectively) [61]. Atrial fibrillation has also been more frequently observed in patients with heart failure and OSA, compared to those with heart failure but without OSA (22\% versus $5 \%$, respectively) [62], and in patients with both hypertrophic cardiomyopathy (HCM) and OSA, compared to those with HCM but without OSA (31\% versus $6 \%$, respectively) [63].
In patients with atrial fibrillation, the prevalence of OSA has been found to be between $21 \%$ and $49 \%[64,65]$, and it is higher in patients with lone atrial fibrillation, compared to matched control subjects with other cardiovascular disease $(49 \%$ versus $32 \%)[64,66]$.

OSA has been suggested to increase the risk for atrial fibrillation recurrence after cardioversion or catheter ablation. In an uncontrolled study of 3000 patients having pulmonary vein isolation (PVI) therapy for atrial fibrillation, $21 \%$ of the study population was diagnosed with OSA [65]. More recently, moderate-to-severe OSA was found to be an independent predictor for atrial fibrillation recurrence in patients undergoing PVI [67].

After an average follow-up period of 32 months, $32 \%$ of the patients without CPAP therapy experienced atrial fibrillation recurrence, while only $21 \%$ of patients with effective CPAP therapy did [65]. Furthermore, in 174 patients who had been treated with catheter ablation therapy for atrial fibrillation, the risk of atrial fibrillation ablation failure has been shown to be independently associated with the severity of OSA [68]. The probability of relapse was $52 \%$ among patients without OSA, and $86 \%$ among patients with severe OSA [68].

Studies regarding the prevalence of atrial fibrillation in patients with OSA are summarised in table 1.

\section{Observational studies regarding atrial arrhythmias}

The prevalence of supraventricular (atrial and sinus) arrhythmias has been estimated to be approximately 50\% among patients with severe OSA, compared with about $25 \%$ in patients with mild OSA and $20 \%$ in control subjects without OSA [71]. In a study looking at patients with newly diagnosed OSA, a prevalence of supraventricular ectopics (SVE) of up to $98 \%$, and SVT of up to $35 \%$, was found [70]. Moreover, patients with OSA were found to have more sinus tachycardia and SVE compared with simple snorers and control subjects without OSA. The number of both nocturnal SVT and SVE has been shown to be significantly higher in patients experiencing nocturnal hypoxia and was related to minimum oxygen saturation during the night $[46,71]$. In one of these studies the incidence of daytime SVT was correlated with increased nocturnal and diurnal urinary catecholamine excretion, and the number of daytime sinus tachycardia were correlated with apnoea/hypopnoea index (AHI) [46].

\section{Interventional studies}

In a small prospective study, recurrence of atrial fibrillation one year after cardioversion was found in $82 \%$ of patients who were not, or were poorly, treated with CPAP compared with $42 \%$ in patients who were effectively treated with CPAP [69]. However, the use of CPAP was not randomised in the latter study, and patients not using CPAP might generally be less compliant with medical therapy, thus potentially introducing a bias. In another uncontrolled study, including 316 patients with newly diagnosed OSA, CPAP therapy significantly reduced the amount of nocturnal paroxysmal atrial fibrillation and SVE from $14 \%$ to $4 \%$ [60].

A randomised-controlled trial (RCT) including patients with newly diagnosed moderate to severe OSA showed no effect of therapeutic CPAP on the frequency of SVE and SVT, when 


\section{TABLE 1 Atrial fibrillation}

\section{First author [ref.] \\ Observational studies}

\section{Study}

design
GuILLEMINAULt [23]

MOOE [61]

OBS

OBS

400

121

JAVAHERI [62]

OBS

81

GAMI [64]

CC

151

PORTHAN [66]

Mehra [10]

GAMI [59]

Pedrosa [63]

OBS

80

59

56

228

338

OBS

3542

Patel [65]

Matiello [68]

OBS

174

ABE [60]

OBS

1350

44

82

BITTER [67]

OBS

INT

39

KanAgala [69]

ABE [60]

Craig [70]
INT

RCT
OSA

Coronary artery bypass surgery

Males, heart failure with LVEF < $45 \%$

Patients undergoing electroversion for $\mathrm{AF}$

Controls referred to cardiologist, no AF Patients with AF Controls without $A F$ Patients with SDB (RDI $\geqslant 30$ events $\cdot h^{-1}$ ) Controls without SDB (RDI $<5$ events $\cdot h^{-1}$ )

Obese subjects

Hypertrophic cardiomyopathy

PVAl for AF

Circumferential pulmonary vein ablation for $A F$

OSA no SDB
Patients undergoing pulmonary vein ablation for $\mathrm{AF}$

Patients with AF and OSA Controls with only AF

OSA no SDB

OSA therapeutic CPAP versus sub-therapeutic CPAP
Main findings

$3 \%$ prevalence of nocturnal paroxysmal AF

AF incidence postoperatively: $32 \%$ for $A H I \geqslant 5$ events $\cdot h^{-1}$, $18 \%$ for $\mathrm{AHI}<5$ events $\cdot \mathrm{h}^{-1}$,

$39 \%$ for ODI $\geqslant 5$ events $\cdot h^{-1}, 18 \%$ for ODI $<5$ events $\cdot h^{-1}$

$\mathrm{ODI}$ independent predictor of post-operative $\mathrm{AF}$ onset $11 \%$ prevalence of OSA

$22 \%$ prevalence of AF among patients with SDB with a four-fold increase in relative risk

$49 \%$ prevalence of OSA in patients with $\mathrm{AF}$ OR 2.19 for risk of AF in OSA patients

$32 \%$ prevalence of OSA in patients with AF

Neck circumference is independently associated with AF in males $4.8 \%$ versus $0.9 \%$ prevalence of $\mathrm{AF}$

OR 4.02 for AF after adjusting for age, sex, body mass index coronary artery disease

$74 \%$ prevalence of OSA (for $\mathrm{AHI} \geqslant 5$ events $\cdot \mathrm{h}^{-1}$ ) Incidence of $\mathrm{AF}$ at 5-year follow-up: $4.3 \%$ in patients with OSA, $2.1 \%$ in patients without OSA HR 2.18 for AF in patients with OSA

$40 \%$ prevalence of OSA, 31\% prevalence of AF in OSA patients, $6 \%$ in patients without OSA OSA and left atrial diameter are independently associated with AF

$21.3 \%$ prevalence of OSA $27 \%$ incidence of AF recurrence after treatment in OSA patients

OSA independently associated with failure of PVAI $24.2 \%$ prevalence of OSA

Incidence of AF recurrence after treatment: $51.5 \%$ for $\mathrm{AHI}<10$ events $\cdot \mathrm{h}^{-1}$, $85.7 \%$ for $\mathrm{AHI} \geqslant 30$ events $\cdot h^{-1}$

Prevalence of paroxysmal AF during polysomnography: $1 \%$ for AHI 5-15 events $\cdot h^{-1}, 3.3 \%$ for AHI 15-30 events $\cdot h^{-1}$, $3.4 \%$ for $\mathrm{AHI} \geqslant 30$ events $\cdot \mathrm{h}^{-1}$

Prevalence of chronic AF: $2.3 \%$ for $\mathrm{AHI}<5$ events $\cdot h^{-1}, 3.6 \%$ for AHI 5-15 events $\cdot h^{-1}, 5.2 \%$ for AHI 15-30 events $\cdot h^{-1}$, $3.8 \%$ for $\mathrm{AHI}>30$ events. $h^{-1}$

Prevalence of paroxysmal AF: $2.3 \%$ for $\mathrm{AHI}<5$ events. $\mathrm{h}^{-1}, 7.6 \%$ for AHI 5-15 events. $h^{-1}, 5.2 \%$ for AHI 15-30 events. $h^{-1}$, $6.6 \%$ for $\mathrm{AHI}>30$ events. $h^{-1}$

$20 \%$ prevalence of moderate to severe OSA AF recurrence $45.5 \%$ for moderate to severe SDB, $24.5 \%$ for mild or no SDB

Recurrence of AF 12 months after cardioversion: 82\% in OSA patients without CPAP therapy, $42 \%$ in OSA patients with CPAP therapy, $53 \%$ in control subjects

CPAP treatment significantly reduced nocturnal paroxysmal AF and supraventricular ectopies from $14 \%$ to $4 \%$ CPAP does not affect the frequency of atrial arrhythmias

OBS: observational study; OSA: obstructive sleep apnoea; AF: atrial fibrillation; AHI: apnoea/hypopnoea index; ODI: oxygen desaturation index; LVEF: left ventricular ejection fraction; SDB: sleep disordered breathing; CC: case-control study; RDI: respiratory disturbance index; PVAI: pulmonary vein antrum isolation; INT: interventional uncontrolled study; CPAP: continuous positive airway pressure; RCT: randomised controlled trial. 
compared to patients on sub-therapeutic CPAP [70]. However, the latter study was not adequately powered to definitely answer the question of whether CPAP improves the frequency of SVE and SVT. Interventional studies on the effect of CPAP on atrial fibrillation in patients with OSA are summarised in table 1.

Due to the lack of appropriately powered RCTs, specifically looking at the effect of CPAP on atrial fibrillation and supraventricular arrhythmias, there is currently very little robust evidence to support the hypothesis that OSA is causally related to atrial fibrillation and supraventricular arrhythmias. Further RCTs investigating the effects of CPAP on the occurrence of atrial fibrillation and supraventricular arrhythmias, as well as RCTs looking at the recurrence rates of atrial fibrillation after cardioversion, are urgently needed.

\section{Bradycardia, sinus pauses and atrio-ventricular blocks \\ Observational studies}

Bradycardia, with a reported prevalence of between $8 \%$ and $95 \%$, is commonly found in patients with OSA [72, 73], and the occurrence of bradycardia seems to be related to the extent of hypoxaemia [26]. Bradyarrhythmias, such as atrio-ventricular block, sinus pause and asystole, occur in up to $18 \%$ of patients with OSA, even in absence of cardiac conduction disease [23, $25,26,72,74]$. In comparison, in a healthy elderly population aged between 60 and 85 years, the prevalence of nocturnal bradyarrhythmias was only $3 \%$ [75]. In the European multicentre polysomnographic study, $58 \%$ of patients implanted with a pacemaker for sinus node dysfunction, and $68 \%$ of those treated with a pacemaker for atrio-ventricular block, had minimally symptomatic OSA and $27 \%$ fulfilled the criteria for severe OSA [76].

It has been proposed that the incidence of bradycardic arrhythmias depends on the severity of OSA, and has been observed in about $8 \%$ of patients diagnosed with an AHI $<60$ events $\cdot h^{-1}$ and in about $20 \%$ of patients with very severe OSA $\left(\right.$ AHI $>60$ events $\left.\cdot h^{-1}\right)$ [73]. Another study showed similar results, with a prevalence of bradyarrhythmias of $8 \%$ in OSA patients with an AHI $>30$ events $\cdot h^{-1}$ compared with $2 \%$ in patients with an AHI $<30$ events $\cdot h^{-1}$ [11]. Atrio-ventricular blocks, primarily grade II and III, are generally registered during rapid eye movement (REM) sleep when apnoeas are typically longer and oxygen desaturation is more pronounced, and have been reported to occur in about $10 \%$ of patients with OSA, compared with $1 \%$ in the healthy elderly population [23, $73,75]$. Studies reporting the prevalence of bradyarrhythmias in patients with OSA are summarised in table 2.

\section{Interventional studies}

In an early uncontrolled study including 15 patients with OSA and severe bradycardia (heart rate $<30$ beats $\cdot \mathrm{min}^{-1}$ ), or asystoles of 2.5 to $3.6 \mathrm{~s}$ or second-degree atrio-ventricular block, atropine was partially effective and treatment of OSA by tracheostomy was highly effective, in preventing these arrhythmias during sleep [72]. Sinus pauses are suspected to be longer in OSA patients compared with age-matched controls, and seem to be prevented by treatment with CPAP in $80-90 \%$ of patients $[73,75]$. Moreover, CPAP seems to be effective in preventing both cardiac pauses of $>3 \mathrm{~s}$ duration and bradycardic episodes $\left(<40\right.$ beats $\left.\cdot \mathrm{min}^{-1}\right)$, as the frequency of these dysrhythmias was significantly decreased at 8 weeks; and they completely disappeared after 6 months of CPAP treatment in two uncontrolled interventional studies [25, 77]. In contrast, in a randomised and controlled study including patients with newly diagnosed moderate-to-severe OSA, CPAP was not associated with a reduction of bradycardic episodes [70]. However, this discordant finding may be due to differences in the study populations or in treatment times [70]. Studies describing the effect of OSA treatment on bradyarrhythmias are summarised in table 2.

In summary, to date there is preliminary evidence supporting the hypothesis that OSA is causally associated with bradyarrhythmias. However, currently there are no data from RCTs proving that CPAP is an effective therapy to reduce the number of both cardiac pauses and bradycardic episodes. This issue will need to be clarified in interventional RCTs including a carefully selected population of OSA patients with frequent bradycardic episodes.

\section{Ventricular repolarisation disturbances}

In vitro studies using myocardial cells have identified several electrocardiographic correlates of ventricular electrical activity that are thought to reflect the risk for the onset of ventricular dysrhythmias [78]. The most important and commonly analysed indices are the QT interval, the QT dispersion, and the Tpeak to Tend (TpTe) interval.

The QT interval is the electrocardiographic representation of the ventricular depolarisation and repolarisation, including the vulnerable period for re-entry to tachycardia (fig. 2) [74, 79]. The QT dispersion is the difference between the maximal and the minimal QT interval length within the same 12-lead ECG recording and is thought to represent the different local vectors of repolarisation, thus creating different T-loops on the surface ECG [80]. Several studies have identified inherited as well as acquired QT prolongation as a risk factor for the occurrence of malignant cardiac dysrhythmias and sudden cardiac death (SCD) [74, 79]. In a prospective analysis of the populationbased Rotterdam study, it has been demonstrated that those with the highest QTc tertile had a 2.5-times higher risk for any cardiac death and a 1.9-times higher risk for sudden cardiac death [80].

The TpTe is a measure of cardiac transmural dispersion of repolarisation, which is explained by a gradient of action potential duration from subendocardial $\mathrm{M}$ cells (longest) to epicardial cells (shortest) (fig. 2) [78, 81]. A prolonging of the TpTe interval to $>100 \mathrm{~ms}$ is proposed to be associated with increased vulnerability for the occurrence of early after-depolarisations and, thus, ventricular tachycardia and SCD [82-84].

\section{Observational studies}

In patients with OSA, the QT interval (corrected for heart rate; QTc) has been found to be considerably prolonged at the onset of the apnoea and to shorten during the post-apnoea hyperventilation period [85]. Furthermore, in patients with OSA, QT dynamicity, a marker of augmented myocardial vulnerability to arrhythmias, has been found to be related to the severity of AHI [86]. 


\section{TABLE 2 Bradyarrhythmias}

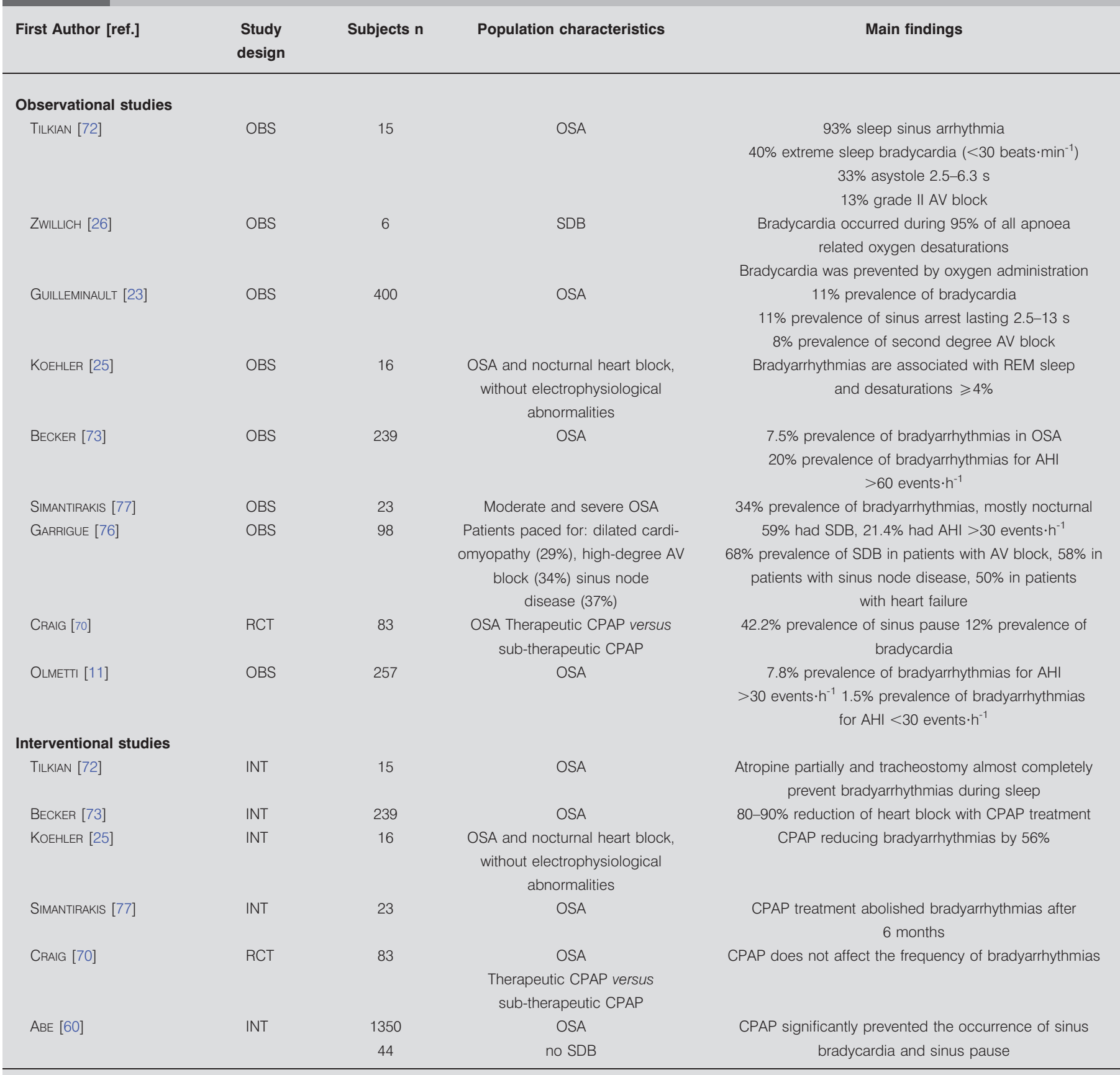

OBS: observational study; OSA: obstructive sleep apnoea; AV: atrio-ventricular; SDB: sleep disordered breathing; REM: rapid eye movement; AHI: apnoea/hypopnoea index; RCT: randomised controlled trial; CPAP: continuous positive airway pressure; INT: interventional uncontrolled study.

\section{Interventional studies}

In a small uncontrolled study, repolarisation alterations were registered mainly during non-REM sleep and disappeared after CPAP therapy, thus suggesting a causal role played by OSA [85]. Moreover, OSA might lead to abnormal ventricular repolarisation, characterised by a flattened relationship between QT duration and heart rate; this finding reflects alterations in the autonomic function and seems also be reversible by CPAP treatment $[87,88]$.
In a randomised-controlled trial, analysis of QTc and TpTec (TpTe corrected for heart rate) intervals from a 12-lead ECG found that CPAP withdrawal for 2 weeks was associated with both prolongation, and an increase in dispersion, of repolarisation, providing a possible mechanistic link between OSA, cardiac arrhythmias and SCD [89]. The increase in the length of these intervals was positively correlated with the change in the severity of OSA, suggesting that the risk for malignant dysrhythmias increases with the severity of OSA. In addition, 
a)

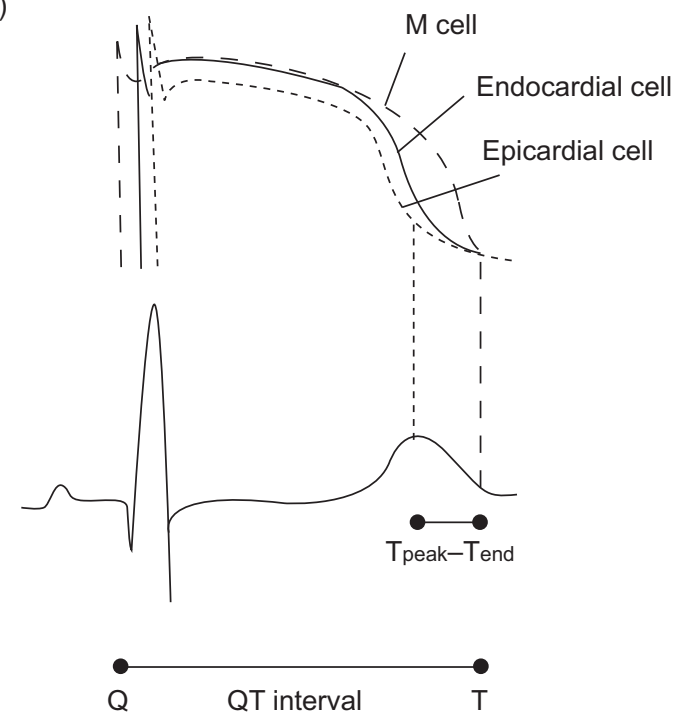

b)

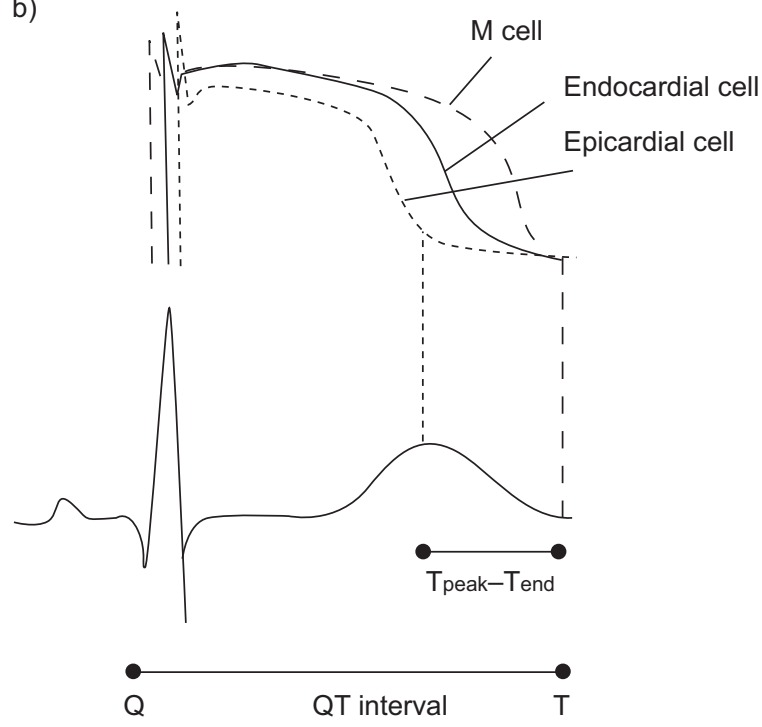

FIGURE 2. Measures of cardiac repolarisation. a) Homogeneous myocardium and b) heterogeneous myocardium. The QT interval is the sum of the ventricular depolarisation (QRS complex) and the ventricular repolarisation (ST segment and T wave). A prolongation of this interval has been associated with increased risk of ventricular dysrhythmias and sudden cardiac death (SCD). The Tpeak to Tend interval represents the dispersion of the ventricular repolarisation. The Tpeak represents the repolarisation of the epicardial cells, while the Tend represents the repolarisation of the subendocardial $\mathrm{M}$ cells, which have longer repolarisation periods and therefore are more susceptible to early after-depolarisation. An increase in the length of this interval is a measure of electrical heterogeneity within the ventricular myocardium thus increasing the risk for ventricular dysrhythmias and SCD.

there was a correlation between the change in the QTc and change in the urinary noradrenaline levels, suggesting that increased sympathetic nervous system activity may be one of the underlying mechanisms between OSA and the increased dispersion of cardiac repolarisation [89]. According to PANIKKATH et al. [83] an uncorrected TpTe interval $>100 \mathrm{~ms}$, and a QTc interval $>430 \mathrm{~ms}$ in the resting ECG, are associated with an increased risk of SCD. Using these thresholds in the latter randomised controlled trial, more than $50 \%$ of patients with OSA were found to be at increased risk for SCD after 2 weeks of CPAP therapy withdrawal [89].

Thus, there is strong RCT evidence showing that OSA is associated with cardiac repolarisation disturbances, and CPAP seems to be effective in normalising cardiac repolarisation in patients who are highly compliant with CPAP. However, future interventional trials will need to address the question as to whether long-standing OSA is associated with chronic and irreversible alterations of cardiac repolarisation. Due to the ethical issues related to a long-term control group, it will be very difficult to perform RCTs to clarify this point.

\section{Ventricular arrhythmias}

Observational studies

In patients with OSA, the prevalence of nocturnal ventricular premature complexes (VPCs) has been found to be between $14 \%$ and $74 \%$, whereas VPCs have been estimated to occur in about $5 \%$ of the general population [1, 23, 24, 72]. The wide range of the reported prevalence may be explained by different OSA severities in the studied populations. Recent findings from the Sleep Heart Health Study, based on one night of monitoring, suggest that subjects with severe OSA have a prevalence of $5 \%$ for non-sustained ventricular tachycardia and of $25 \%$ for complex ventricular ectopy, such as bigeminy, trigeminy or quadrigeminy, with an odds ratio of 3.4 and 1.8, respectively, when compared with the general population [10]. VPCs were observed mainly during the night at the end of apnoeas, and when oxygen desaturations were most pronounced [90]. Recently, two studies investigated the time of VPCs occurrence during the night and revealed an increased onset during the apnoeic phase and shortly after events of disturbed breathing when compared with normal breathing periods, thus supporting possible direct effects of OSA on the inducibility of arrhythmias [91, 92].

Similar results were observed in a prospective study, where $58 \%$ of patients with an $\mathrm{AHI}>10$ events $\cdot \mathrm{h}^{-1}$, and $82 \%$ of patients with mean nocturnal oxygenation $<90 \%$, had supraand ventricular ectopics [71]. In a study including 38 patients with reduced left ventricular ejection fraction treated with a cardioverter-defibrillator, $41 \%$ had sleep-related breathing disorders (OSA and Cheyne-Stokes breathing) and VPCs occurred significantly more often during sleep disordered breathing than during normal respiration [93]. KOSHINO et al. [90] found that $60 \%$ of all patients with ventricular arrhythmias referred for catheter ablation or implantation of a cardioverterdefibrillator met the criteria for OSA (AHI $\geqslant 5$ events $\cdot \mathrm{h}^{-1}$ ), and $34 \%$ met the criteria for moderate-severe OSA (AHI $\geqslant 15$ events $\left.\cdot h^{-1}\right)$.

In a larger study, including 472 patients with congestive heart failure, 32\% were found to have untreated OSA and 34\% had CSA. After a 48 month follow-up period, sleep disordered breathing was found to be independently associated with an increased risk for ventricular arrhythmias [94]. Moreover, OSA patients treated with catheter ablation for ventricular dysrhythmias seem to have a higher relapse rate compared to 
those without OSA ( $45 \%$ versus $6 \%$ ) [95]. In a case-control study, comparison of the occurrence rate of VPCs between patients with OSA, simple snorers and healthy subjects showed that patients with OSA have a significantly higher rate of VPCs than control subjects, which seems to be related to increased sympathetic activation [46]. However, this is in contrast to MiLLER [96] who reviewed $24 \mathrm{~h}$ Holter ECGs of 23 patients with OSA, and found that the prevalence of VPCs and ventricular arrhythmias during sleep is not different when compared to wakefulness [96]. In a more recent study, ECG data from 257 patients with newly diagnosed OSA were analysed [11]. Only 9\% of patients had VPCs and no correlation with OSA severity was found [11]. Because of these conflicting findings it is uncertain whether ventricular arrhythmias are more frequently observed in patients with OSA than in comparable populations. The studies describing the prevalence of ventricular arrhythmias in patients with OSA and vice versa are summarised in table 3.

\section{Interventional studies}

In an uncontrolled study including 15 patients with OSA, nocturnal VPCs and episodes of ventricular tachycardia could be partially resolved after atropine administration, and almost completely abolished after tracheostomy, suggesting a possible role of OSA in eliciting these arrhythmias [72].

In a randomised-controlled study including 18 patients with OSA and heart failure, CPAP therapy for 1 month reduced the number of VPCs by $58 \%$ when compared to patients without CPAP therapy, and this effect was related to a reduction of urinary noradrenaline excretion [98]. In another randomisedcontrolled study, which included 83 patients with moderatesevere OSA, $24 \mathrm{~h}$ Holter monitoring was performed before and after 1 month of therapeutic or sub-therapeutic CPAP therapy [70]. The authors did not find any statistically significant change for any ventricular arrhythmia during night or day, although a trend towards less daytime ventricular tachycardia was observed in the therapeutic CPAP group. However, the interpretability of this study is limited by the relative small number of patients with arrhythmias included [70]. Interventional studies regarding the association between ventricular arrhythmias and OSA are summarised in table 3.

Due to the very limited data available from RCTs, it is currently not known whether there is a causal relationship between OSA and ventricular arrhythmias. To date, there is also no evidence proving that CPAP is an effective therapy to re-establish a normal heart rhythm in OSA patients with ventricular arrhythmias. Thus, there is a need for well-designed and appropriately powered controlled trials evaluating the impact of CPAP on ventricular arrhythmias in patients with OSA.

\section{SUDDEN CARDIAC DEATH}

Evidence from observational data suggests that in patients with OSA, the onset of myocardial infarction occurs more frequently during the night, compared to subjects without OSA (32\% versus $7 \%$ ) [99]. Moreover, patients who experienced myocardial infarction during the night had an odds ratio of 6.0 of having OSA [99]. In patients with OSA, malignant rhythm alterations, such as ventricular asystole up to $13 \mathrm{~s}$, ventricular tachycardia and fibrillation, were recorded during the night and were related to AHI severity $[23,77,97]$. CPAP therapy seems to have a protective effect against cardiac adverse events. Evidence from interventional studies demonstrated that CPAP therapy was effective in reducing severe arrhythmic events in $87 \%$ of cases [97]. Moreover, patients with OSA, non-compliant with CPAP therapy, were more susceptible to cardiovascular deaths and events, compared to CPAP compliant patients (15\% versus $2 \%$ and $31 \%$ versus $18 \%$, respectively) $[97,100]$.

In a retrospective observational study, GAMI et al. [8] reviewed polysomnographic studies and death certificates from 112 subjects with SCD and found that $46 \%$ of the subjects diagnosed with OSA died between midnight and 06:00 h while only $21 \%$ of the subjects without OSA died during the night. This compares to $16 \%$ in the general population and to $25 \%$ as expected by chance. Similarly, subjects who died during the night had a higher prior AHI compared to those who died during daytime [8]. Compared to subjects without OSA, the relative risk of SCD between midnight and 06:00 $\mathrm{h}$ was 2.6 (95\% CI 1.9-3.5) for patients with OSA. Although the retrospective design of this study does not allow a causal relationship between OSA and SCD to be established, the findings suggest that OSA might be a risk factor for SCD. However, due to the ethical concerns of withholding CPAP treatment for longer periods, it is very unlikely that there will ever be data from a randomised controlled interventional trial which has been specifically designed and appropriately powered to prove this relationship.

\section{CLINICAL IMPLICATIONS}

The potential clinical importance of the different cardiac arrhythmias observed in patients with OSA is highly variable, i.e. occasional single VPCs and short episodes of nocturnal bradycardia may not impact on a patient's well-being or prognosis, whereas symptomatic atrial fibrillation, disturbed repolarisation and malignant ventricular arrhythmia may be associated with increased morbidity and mortality. In addition, the clinical implications of cardiac arrhythmias also depend on the comorbidities in patients with OSA; i.e. patients with concomitant cardiovascular disease may have worse outcomes. As there is currently no definitive proof that CPAP is an effective therapy to re-establish a normal heart rhythm in patients with OSA and arrhythmia, additional arrhythmiaspecific treatment should be considered in these patients, particularly since CPAP may not be worn every night. The decision whether anti-arrhythmic therapy should be initiated, depends on the aetiology and mechanism of the arrhythmia, as well as on the patient's symptoms, characteristics and comorbidities, and should follow international guidelines (www. escardio.org) [101, 102]. As such, patients with atrial fibrillation, for example, may require antithrombotic therapy, oral antiarrhythmic drug therapy, cardioversion or ablation therapy [101]. Similarly, patients with ventricular arrhythmia who are often symptomatic may become haemodynamically unstable and are at a high risk for SCD. Treatment should, therefore, include specific antiarrhythmic drugs, implantable devices, ablation and surgery [102]. Clearly such patients should be referred to a specialised cardiologist for further evaluation and treatment.

\section{CONCLUSIONS}

Several mechanisms are thought to underpin the association between OSA and cardiac arrhythmias: intermittent hypoxia associated with both autonomic nervous system activation and 
TABLE 3 Ventricular arrhythmias

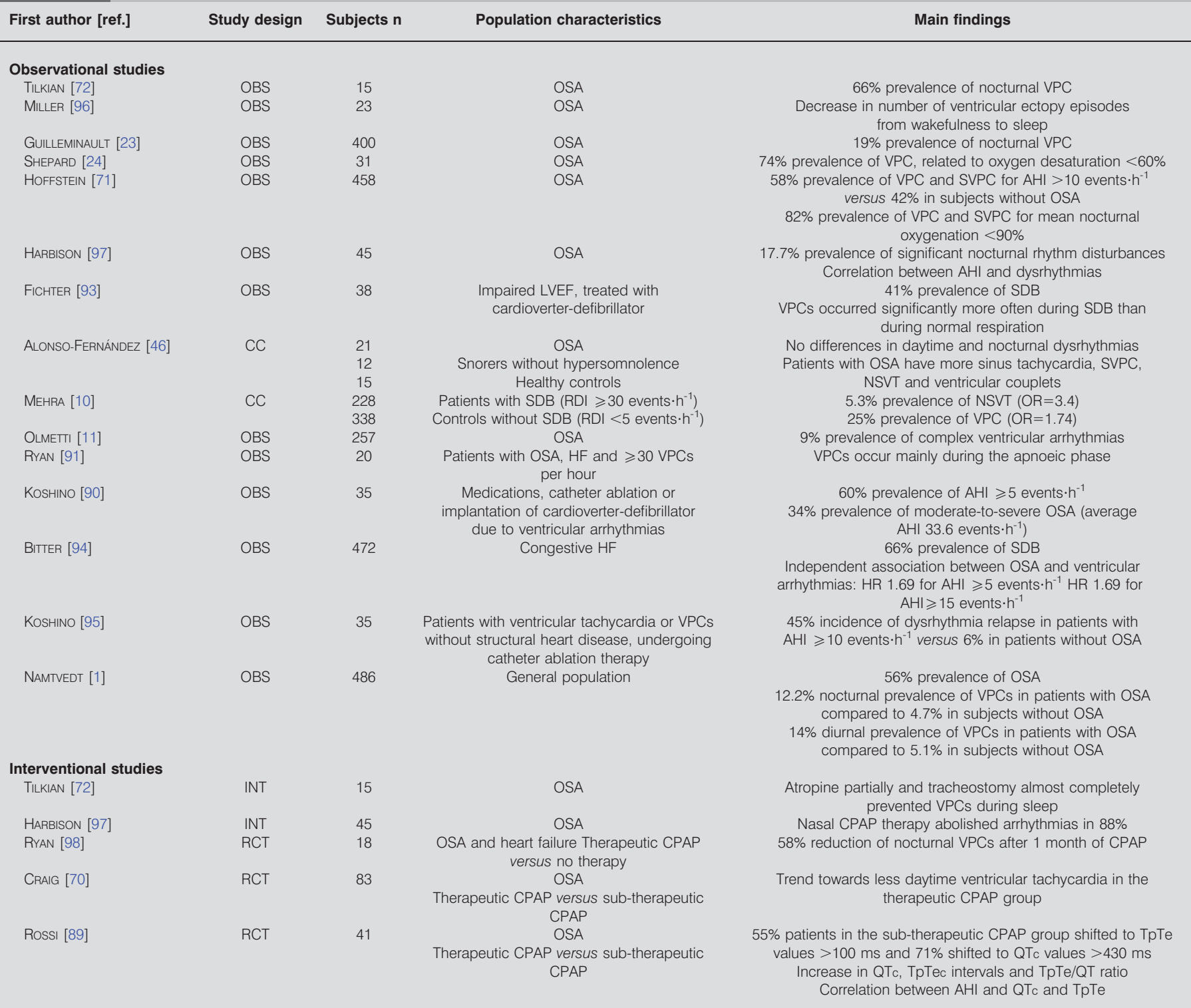

OBS: observational study; OSA: obstructive sleep apnoea; VPC: ventricular premature complex; SVPC: supraventricular premature complex; AHI: apnoea/hypopnoea index; LVEF: left ventricular ejection fraction; SDB: sleep disordered breathing; CC: case-control study; NSVT: non-sustained ventricular tachycardia; RDI: respiratory disturbance index; HF: heart failure; INT: interventional uncontrolled study; CPAP: continuous positive airway pressure; RCT: randomised controlled study; TpTe: Tpeak to Tend interval; QTc: QT interval corrected for heart rate; TpTec: TpTe corrected for heart rate.

increased oxidative stress, the latter potentially leading to cardiac cellular damage and alteration in myocardial excitability; recurrent arousals, resulting in sympathetic activation and coronary vasoconstriction; and increased negative intrathoracic pressure which may mechanically stretch the myocardial walls and thus promote acute changes in myocardial excitability as well as structural remodelling of the myocardium.

Findings from cross-sectional studies suggest both a high prevalence of cardiac arrhythmia in patients with OSA, and a high prevalence of OSA in those with cardiac arrhythmia. The findings of observational studies also support the hypothesis that OSA may be a causal factor for SCD, which may result from malignant cardiac arrhythmias or acute ischaemic heart disease. Preliminary evidence from interventional studies suggests that treatment of OSA may reduce cardiac arrhythmias. However, there is very little data from randomised controlled trials on this topic and future research in patients with OSA should address this important issue.

\section{STATEMENT OF INTEREST}

None declared.

\section{REFERENCES}

1 Namtvedt SK, Randby A, Einvik G, et al. Cardiac arrhythmias in obstructive sleep apnea (from the Akershus sleep apnea project). Am J Cardiol 2011; 108: 1141-1146. 
2 Stradling JR, Crosby JH. Predictors and prevalence of obstructive sleep apnoea and snoring in 1001 middle aged men. Thorax 1991; 46: 85-90.

3 Young T, Peppard PE, Gottlieb DJ. Epidemiology of obstructive sleep apnea: a population health perspective. Am J Respir Crit Care Med 2002; 165: 1217-1239.

4 Pepperell JC, Ramdassingh-Dow S, Crosthwaite N, et al. Ambulatory blood pressure after therapeutic and subtherapeutic nasal continuous positive airway pressure for obstructive sleep apnoea: a randomised parallel trial. Lancet 2002; 359: 204-210.

5 Kohler M, Stradling JR. Mechanisms of vascular damage in obstructive sleep apnea. Nat Rev Cardiol 2010; 7: 677-685.

6 Marin JM, Carrizo SJ, Vicente E, et al. Long-term cardiovascular outcomes in men with obstructive sleep apnoea-hypopnoea with or without treatment with continuous positive airway pressure: an observational study. Lancet 2005; 365: 1046-1053.

7 Somers VK, White DP, Amin R, et al. Sleep apnea and cardiovascular disease: an American Heart Association/American College Of Cardiology Foundation Scientific Statement from the American Heart Association Council for High Blood Pressure Research Professional Education Committee, Council on Clinical Cardiology, Stroke Council, and Council On Cardiovascular Nursing. In collaboration with the National Heart, Lung, and Blood Institute National Center on Sleep Disorders Research (National Institutes of Health). Circulation 2008; 118: 1080-1111.

8 Gami AS, Howard DE, Olson EJ, et al. Day-night pattern of sudden death in obstructive sleep apnea. N Engl J Med 2005; 352 1206-1214.

9 Cortelli $\mathrm{P}$, Lombardi C, Montagna P, et al. Baroreflex modulation during sleep and in obstructive sleep apnea syndrome. Auton Neurosci 2012; 169: 7-11.

10 Mehra R, Benjamin EJ, Shahar E, et al. Association of nocturnal arrhythmias with sleep-disordered breathing: The Sleep Heart Health Study. Am I Respir Crit Care Med 2006; 173: 910-916.

11 Olmetti F, La Rovere MT, Robbi E, et al. Nocturnal cardiac arrhythmia in patients with obstructive sleep apnea. Sleep Med 2008; 9: 475-480.

12 Leung RS, Huber MA, Rogge T, et al. Association between atrial fibrillation and central sleep apnea. Sleep 2005; 28: 1543-1546.

13 Camen G, Clarenbach CF, Stöwhas AC, et al. The effects of simulated obstructive apnea and hypopnea on arrhythmic potential in healthy subjects. Eur J Appl Physiol 2013; 113: 489-496.

14 De Daly MB, Scott MJ. The effects of stimulation of the carotid body chemoreceptors on heart rate in the dog. J Physiol 1958; 144 148-166.

15 Daly MD, Scott MJ. The cardiovascular responses to stimulation of the carotid body chemoreceptors in the dog. J Physiol 1963; 165: 179-197.

16 Peng YJ, Yuan G, Ramakrishnan D, et al. Heterozygous HIF-1 $\alpha$ deficiency impairs carotid body-mediated systemic responses and reactive oxygen species generation in mice exposed to intermittent hypoxia. J Physiol 2006; 577: 705-716.

17 Mody P, Rukhadze I, Kubin L. Rats subjected to chronicintermittent hypoxia have increased density of noradrenergic terminals in the trigeminal sensory and motor nuclei. Neurosci Lett 2011; 505: 176-179.

18 Rukhadze I, Fenik VB, Benincasa KE, et al. Chronic intermittent hypoxia alters density of aminergic terminals and receptors in the hypoglossal motor nucleus. Am J Respir Crit Care Med 2010; 182: 1321-1329.

19 Souvannakitti D, Kumar GK, Fox A, et al. Contrasting effects of intermittent and continuous hypoxia on low $\mathrm{O}_{2}$ evoked catecholamine secretion from neonatal rat chromaffin cells. Adv Exp Med Biol 2009; 648: 345-349.

20 Ghias M, Scherlag BJ, Lu Z, et al. The role of ganglionated plexi in apnea-related atrial fibrillation. J Am Coll Cardiol 2009; 54: 2075-2083.
21 Hedner J, Ejnell H, Sellgren J, et al. Is high and fluctuating muscle nerve sympathetic activity in the sleep apnoea syndrome of pathogenetic importance for the development of hypertension? J Hypertens Suppl 1988; 6: S529-S531.

22 Hla KM, Young TB, Bidwell $\mathrm{T}$, et al. Sleep apnea and hypertension. A population-based study. Ann Intern Med 1994; 120: 382-388.

23 Guilleminault C, Connolly SJ, Winkle RA. Cardiac arrhythmia and conduction disturbances during sleep in 400 patients with sleep apnea syndrome. Am J Cardiol 1983; 52: 490-494.

24 Shepard JW Jr, Garrison MW, Grither DA, et al. Relationship of ventricular ectopy to oxyhemoglobin desaturation in patients with obstructive sleep apnea. Chest 1985; 88: 335-340.

25 Koehler U, Fus E, Grimm W, et al. Heart block in patients with obstructive sleep apnoea: pathogenetic factors and effects of treatment. Eur Respir J 1998; 11: 434-439.

26 Zwillich C, Devlin T, White D, et al. Bradycardia during sleep apnea. Characteristics and mechanism. J Clin Invest 1982; 69: 1286-1292.

27 Kohler M, Stoewhas AC, Ayers L, et al. Effects of continuous positive airway pressure therapy withdrawal in patients with obstructive sleep apnea: a randomized controlled trial. Am J Respir Crit Care Med 2011; 184: 1192-1199.

28 Kohler M, Pepperell JC, Casadei B, et al. CPAP and measures of cardiovascular risk in males with OSAS. Eur Respir I 2008; 32: 1488-1496.

29 Prabhakar NR, Kumar GK. Oxidative stress in the systemic and cellular responses to intermittent hypoxia. Biol Chem 2004; 385: 217-221.

30 Peng Y, Yuan G, Overholt JL, et al. Systemic and cellular responses to intermittent hypoxia: evidence for oxidative stress and mitochondrial dysfunction. Adv Exp Med Biol 2003; 536: 559-564.

31 Chen L, Einbinder E, Zhang Q, et al. Oxidative stress and left ventricular function with chronic intermittent hypoxia in rats. Am J Respir Crit Care Med 2005; 172: 915-920.

32 Park AM, Suzuki YJ. Effects of intermittent hypoxia on oxidative stress-induced myocardial damage in mice. J Appl Physiol 2007; 102: 1806-1814.

33 Chen L, Zhang J, Gan TX, et al. Left ventricular dysfunction and associated cellular injury in rats exposed to chronic intermittent hypoxia. J Appl Physiol 2008; 104: 218-223.

34 Liu JN, Zhang JX, Lu G, et al. The effect of oxidative stress in myocardial cell injury in mice exposed to chronic intermittent hypoxia. Chin Med J (Engl) 2010; 123: 74-78.

35 Jeong EM, Liu M, Sturdy M, et al. Metabolic stress, reactive oxygen species, and arrhythmia. J Mol Cell Cardiol 2012; 52: 454-463.

36 Cortassa S, Aon MA, Marbán E, et al. An integrated model of cardiac mitochondrial energy metabolism and calcium dynamics. Biophys J 2003; 84: 2734-2755.

37 Brown DA, O'Rourke B. Cardiac mitochondria and arrhythmias. Cardiovasc Res 2010; 88: 241-249.

38 Kumar GK, Rai V, Sharma SD, et al. Chronic intermittent hypoxia induces hypoxia-evoked catecholamine efflux in adult rat adrenal medulla via oxidative stress. J Physiol 2006; 575: 229-239.

39 Alonso-Fernández A, Garcia-Rio F, Arias MA, et al. Effects of CPAP on oxidative stress and nitrate efficiency in sleep apnoea: a randomised trial. Thorax 2009; 64: 581-586.

40 Horner RL, Brooks D, Kozar LF, et al. Immediate effects of arousal from sleep on cardiac autonomic outflow in the absence of breathing in dogs. J Appl Physiol 1995; 79: 151-162.

41 Pinto JM, Garpestad E, Weiss JW, et al. Hemodynamic changes associated with obstructive sleep apnea followed by arousal in a porcine model. J Appl Physiol 1993; 75: 1439-1443.

42 Somers VK, Dyken ME, Mark AL, et al. Sympathetic-nerve activity during sleep in normal subjects. N Engl J Med 1993; 328: 303-307. 
43 Somers VK, Mark AL, Zavala DC, et al. Contrasting effects of hypoxia and hypercapnia on ventilation and sympathetic activity in humans. J Appl Physiol 1989; 67: 2101-2106.

44 Verrier RL, Dickerson LW. Autonomic nervous system and coronary blood flow changes related to emotional activation and sleep. Circulation 1991; 83: II81-II89.

45 Andreas S, von Breska B, Schaumann A, et al. Obstructive sleep apnoea and signal averaged electrocardiogram. Eur Respir J 1995; 8: 546-550.

46 Alonso-Fernández A, García-Río F, Racionero MA, et al. Cardiac rhythm disturbances and ST-segment depression episodes in patients with obstructive sleep apnea-hypopnea syndrome and its mechanisms. Chest 2005; 127: 15-22.

47 Chan KH, Wilcox I. Obstructive sleep apnea: novel trigger and potential therapeutic target for cardiac arrhythmias. Expert Rev Cardiovasc Ther 2010; 8: 981-994.

48 Dimitri $\mathrm{H}, \mathrm{Ng} \mathrm{M}$, Brooks AG, et al. Atrial remodeling in obstructive sleep apnea: implications for atrial fibrillation. Heart Rhythm 2012; 9: 321-327.

49 Tsai CT, Chiang FT, Tseng CD, et al. Mechanical stretch of atrial myocyte monolayer decreases sarcoplasmic reticulum calcium adenosine triphosphatase expression and increases susceptibility to repolarization alternans. J Am Coll Cardiol 2011; 58: 2106-2115.

50 Wang Y, Joyner RW, Wagner MB, et al. Stretch-activated channel activation promotes early afterdepolarizations in rat ventricular myocytes under oxidative stress. Am J Physiol Heart Circ Physiol 2009; 296: H1227-H1235.

51 Linz D, Schotten U, Neuberger HR, et al. Negative tracheal pressure during obstructive respiratory events promotes atrial fibrillation by vagal activation. Heart Rhythm 2011; 8: 1436-1443.

52 Virolainen J, Ventilä $\mathrm{M}$, Turto $\mathrm{H}$, et al. Effect of negative intrathoracic pressure on left ventricular pressure dynamics and relaxation. J Appl Physiol 1995; 79: 455-460.

53 Koshino Y, Villarraga HR, Orban M, et al. Changes in left and right ventricular mechanics during the Mueller maneuver in healthy adults: a possible mechanism for abnormal cardiac function in patients with obstructive sleep apnea. Circ Cardiovasc Imaging 2010; 3: 282-289.

54 Stöwhas AC, Namdar M, Biaggi $\mathrm{P}$, et al. The effect of simulated obstructive apnea and hypopnea on aortic diameter and BP. Chest 2011; 140: 675-680.

55 Orban M, Bruce CJ, Pressman GS, et al. Dynamic changes of left ventricular performance and left atrial volume induced by the mueller maneuver in healthy young adults and implications for obstructive sleep apnea, atrial fibrillation, and heart failure. Am J Cardiol 2008; 102: 1557-1561.

56 Linz D, Mahfoud F, Schotten U, et al. Renal sympathetic denervation suppresses postapneic blood pressure rises and atrial fibrillation in a model for sleep apnea. Hypertension 2012; 60: $172-178$.

57 Somers VK, Dyken ME, Skinner JL. Autonomic and hemodynamic responses and interactions during the Mueller maneuver in humans. J Auton Nerv Syst 1993; 44: 253-259.

58 Yagmur J, Yetkin O, Cansel $\mathrm{M}$, et al. Assessment of atrial electromechanical delay and influential factors in patients with obstructive sleep apnea. Sleep Breath 2012; 16: 83-88.

59 Gami AS, Hodge DO, Herges RM, et al. Obstructive sleep apnea, obesity, and the risk of incident atrial fibrillation. J Am Coll Cardiol 2007; 49: 565-571.

60 Abe H, Takahashi M, Yaegashi H, et al. Efficacy of continuous positive airway pressure on arrhythmias in obstructive sleep apnea patients. Heart Vessels 2010; 25: 63-69.

61 Mooe T, Gullsby S, Rabben T, et al. Sleep-disordered breathing: a novel predictor of atrial fibrillation after coronary artery bypass surgery. Coron Artery Dis 1996; 7: 475-478.

62 Javaheri S, Parker TJ, Liming JD, et al. Sleep apnea in 81 ambulatory male patients with stable heart failure. Types and their prevalences, consequences, and presentations. Circulation 1998; 97: 2154-2159.

63 Pedrosa RP, Drager LF, Genta PR, et al. Obstructive sleep apnea is common and independently associated with atrial fibrillation in patients with hypertrophic cardiomyopathy. Chest 2010; 137: 1078-1084.

64 Gami AS, Pressman G, Caples SM, et al. Association of atrial fibrillation and obstructive sleep apnea. Circulation 2004; 110 364-367.

65 Patel D, Mohanty P, Di Biase L, et al. Safety and efficacy of pulmonary vein antral isolation in patients with obstructive sleep apnea: the impact of continuous positive airway pressure. Circ Arrhythm Electrophysiol 2010; 3: 445-451.

66 Porthan KM, Melin JH, Kupila JT, et al. Prevalence of sleep apnea syndrome in lone atrial fibrillation: a case-control study. Chest 2004; 125: 879-885.

67 Bitter T, Nölker G, Vogt J, et al. Predictors of recurrence in patients undergoing cryoballoon ablation for treatment of atrial fibrillation: the independent role of sleep-disordered breathing J Cardiovasc Electrophysiol 2012; 23: 18-25.

68 Matiello M, Nadal M, Tamborero D, et al. Low efficacy of atrial fibrillation ablation in severe obstructive sleep apnoea patients Europace 2010; 12: 1084-1089.

69 Kanagala R, Murali NS, Friedman PA, et al. Obstructive sleep apnea and the recurrence of atrial fibrillation. Circulation 2003; 107: 2589-2594.

70 Craig S, Pepperell JC, Kohler M, et al. Continuous positive airway pressure treatment for obstructive sleep apnoea reduces resting heart rate but does not affect dysrhythmias: a randomised controlled trial. J Sleep Res 2009; 18: 329-336.

71 Hoffstein V, Mateika S. Cardiac arrhythmias, snoring, and sleep apnea. Chest 1994; 106: 466-471.

72 Tilkian AG, Guilleminault C, Schroeder JS, et al. Sleep-induced apnea syndrome. Prevalence of cardiac arrhythmias and their reversal after tracheostomy. Am J Med 1977; 63: 348-358.

73 Becker HF, Koehler U, Stammnitz A, et al. Heart block in patients with sleep apnoea. Thorax 1998; 53: Suppl. 3, S29-S32.

74 Elming H, Brendorp B, Kober L, et al. QTc interval in the assessment of cardiac risk. Card Electrophysiol Rev 2002; 6: 289-294.

75 Fleg JL, Kennedy HL. Cardiac arrhythmias in a healthy elderly population: detection by 24-hour ambulatory electrocardiography. Chest 1982; 81: 302-307.

76 Garrigue S, Pépin JL, Defaye P, et al. High prevalence of sleep apnea syndrome in patients with long-term pacing: the European Multicenter Polysomnographic Study. Circulation 2007; 115: 1703-1709.

77 Simantirakis EN, Schiza SI, Marketou ME, et al. Severe bradyarrhythmias in patients with sleep apnoea: the effect of continuous positive airway pressure treatment: a long-term evaluation using an insertable loop recorder. Eur Heart J 2004; 25: 1070-1076.

78 Antzelevitch C. Cellular basis for the repolarization waves of the ECG. Ann NY Acad Sci 2006; 1080: 268-281.

79 Viskin S. Long QT syndromes and torsade de pointes. Lancet 1999; 354: 1625-1633.

80 de Bruyne MC, Hoes AW, Kors JA, et al. QTc dispersion predicts cardiac mortality in the elderly: the Rotterdam study. Circulation 1998; 97: 467-472.

81 Xia Y, Liang Y, Kongstad O, et al. Tpeak-Tend interval as an index of global dispersion of ventricular repolarization: evaluations using monophasic action potential mapping of the epi- and endocardium in swine. J Interv Card Electrophysiol 2005; 14: 79-87.

82 Yamaguchi $\mathrm{M}$, Shimizu $\mathrm{M}$, Ino $\mathrm{H}$, et al. $\mathrm{T}$ wave peak-to-end interval and QT dispersion in acquired long QT syndrome: a new index for arrhythmogenicity. Clin Sci (Lond) 2003; 105: $671-676$ 
83 Panikkath R, Reinier K, Uy-Evanado A, et al. Prolonged Tpeakto-tend interval on the resting ECG is associated with increased risk of sudden cardiac death. Circ Arrhythm Electrophysiol 2011; 4: 441-447.

84 Topilski I, Rogowski O, Rosso R, et al. The morphology of the QT interval predicts torsade de pointes during acquired bradyarrhythmias. J Am Coll Cardiol 2007; 49: 320-328.

85 Gillis AM, Stoohs R, Guilleminault C. Changes in the QT interval during obstructive sleep apnea. Sleep 1991; 14: 346-350.

86 Aytemir K, Deniz A, Yavuz B, et al. Increased myocardial vulnerability and autonomic nervous system imbalance in obstructive sleep apnea syndrome. Respir Med 2007; 101: 1277-1282.

87 Roche F, Gaspoz JM, Court-Fortune I, et al. Alteration of QT rate dependence reflects cardiac autonomic imbalance in patients with obstructive sleep apnea syndrome. Pacing Clin Electrophysiol 2003; 26: 1446-1453.

88 Roche F, Barthélémy JC, Garet M, et al. Continuous positive airway pressure treatment improves the QT rate dependence adaptation of obstructive sleep apnea patients. Pacing Clin Electrophysiol 2005; 28: 819-825.

89 Rossi VA, Stoewhas AC, Camen G, et al. The effects of continuous positive airway pressure therapy withdrawal on cardiac repolarization: data from a randomized controlled trial. Eur Heart J 2012; 33: 2206-2212.

90 Koshino Y, Satoh M, Katayose Y, et al. Association of sleepdisordered breathing and ventricular arrhythmias in patients without heart failure. Am J Cardiol 2008; 101: 882-886.

91 Ryan CM, Juvet S, Leung R, et al. Timing of nocturnal ventricular ectopy in heart failure patients with sleep apnea. Chest 2008; 133: 934-940.

92 Monahan K, Storfer-Isser A, Mehra R, et al. Triggering of nocturnal arrhythmias by sleep-disordered breathing events. J Am Coll Cardiol 2009; 54: 1797-1804.

93 Fichter J, Bauer D, Arampatzis S, et al. Sleep-related breathing disorders are associated with ventricular arrhythmias in patients with an implantable cardioverter-defibrillator. Chest 2002; 122 558-561.

94 Bitter T, Westerheide N, Prinz C, et al. Cheyne-Stokes respiration and obstructive sleep apnoea are independent risk factors for malignant ventricular arrhythmias requiring appropriate cardioverter-defibrillator therapies in patients with congestive heart failure. Eur Heart J 2011; 32: 61-74.

95 Koshino Y, Satoh M, Katayose Y, et al. Sleep apnea and ventricular arrhythmias: clinical outcome, electrophysiologic characteristics, and follow-up after catheter ablation. J Cardiol 2010; 55: 211-216.

96 Miller WP. Cardiac arrhythmias and conduction disturbances in the sleep apnea syndrome. Prevalence and significance. Am J Med 1982; 73: 317-321.

97 Harbison J, O'Reilly P, McNicholas WT. Cardiac rhythm disturbances in the obstructive sleep apnea syndrome: effects of nasal continuous positive airway pressure therapy. Chest 2000; 118: 591-595.

98 Ryan CM, Usui K, Floras JS, et al. Effect of continuous positive airway pressure on ventricular ectopy in heart failure patients with obstructive sleep apnoea. Thorax 2005; 60: 781-785.

99 Kuniyoshi FH, Garcia-Touchard A, Gami AS, et al. Day-night variation of acute myocardial infarction in obstructive sleep apnea. J Am Coll Cardiol 2008; 52: 343-346.

100 Doherty LS, Kiely JL, Swan V, et al. Long-term effects of nasal continuous positive airway pressure therapy on cardiovascular outcomes in sleep apnea syndrome. Chest 2005; 127: 2076-2084.

101 Camm AJ, Lip GY, De Caterina R, et al. 2012 focused update of the ESC Guidelines for the management of atrial fibrillation: an update of the 2010 ESC Guidelines for the management of atrial fibrillation. Developed with the special contribution of the European Heart Rhythm Association. Eur Heart J 2012; 33: 2719-2747.

102 Zipes DP, Camm AJ, Borggrefe M, et al. ACC/AHA/ESC 2006 guidelines for management of patients with ventricular arrhythmias and the prevention of sudden cardiac death-executive summary: a report of the American College of Cardiology/ American Heart Association Task Force and the European Society of Cardiology Committee for Practice Guidelines (Writing Committee to Develop Guidelines for Management of Patients with Ventricular Arrhythmias and the Prevention of Sudden Cardiac Death) Developed in collaboration with the European Heart Rhythm Association and the Heart Rhythm Society. Eur Heart J 2006; 27: 2099-2140. 